\title{
QUASI RIESZ TRANSFORMS, HARDY SPACES AND GENERALISED SUB-GAUSSIAN HEAT KERNEL ESTIMATES
}

\author{
LI CHEN
}

(Received 18 March 2015; first published online 10 August 2015)

2010 Mathematics subject classification: primary 35J05; secondary 35K08, 39A12, 42B20, 42B35, $58 \mathrm{~J} 35,60 \mathrm{~J} 10$.

Keywords and phrases: Riesz transform, Hardy space, metric measure space, graph, heat kernel estimate, Poincaré inequality, Sobolev inequality.

In this thesis, we mainly study Riesz transforms and Hardy spaces associated to operators. These two subjects are closely related to volume growth and heat kernel estimates.

We first study the Riesz transform problem on noncompact Riemannian manifolds and on infinite graphs. It is well known that the Riesz transform is $L^{p}$-bounded on Euclidean spaces for $1<p<\infty$. In 1983, Strichartz [9] asked whether one could extend the $L^{p}$-boundedness of the Riesz transform to noncompact Riemannian manifolds. That is, for which class of noncompact Riemannian manifolds and for which $p \in(1, \infty)$ do we have

$$
\|\nabla f \mid\|_{p} \leq C\left\|\Delta^{1 / 2} f\right\|_{p} \quad \text { for all } f \in C_{0}^{\infty} ?
$$

Many answers to this question have been given; see for example [1, 5]. An estimate of the heat kernel is a key ingredient for the $L^{p}$-boundedness of the Riesz transform. By using techniques involving singular integrals, Coulhon and Duong [5] proved the boundedness of the Riesz transform on $L^{p}(1<p \leq 2)$ for noncompact Riemannian manifolds satisfying the doubling volume condition and the Gaussian heat kernel upper estimate. However, it is not known whether the two conditions are necessary for the $L^{p}$-boundedness of the Riesz transform for $1<p \leq 2$.

In Chapter 1, we consider an arbitrary complete noncompact Riemannian manifold without any volume or heat kernel estimate assumptions. We prove that the quasi Riesz transform $\nabla e^{-\Delta} \Delta^{-\alpha}(0<\alpha<1 / 2)$ is always $L^{p}$-bounded for $1<p \leq 2$. We also

Thesis submitted to the Australian National University (through a cotutelle agreement with Université Paris-Sud) in March 2014; degree approved on 18 July 2014; supervisors: Pascal Auscher and Thierry Coulhon.

(c) 2015 Australian Mathematical Publishing Association Inc. 0004-9727/2015 \$16.00 
show that the Riesz transform on Vicsek manifolds (see [3]) is not $L^{p}$-bounded. This is an improvement of the result of Coulhon and Duong in [6].

In Chapter 2, we assume that the manifold satisfies the doubling volume property and the sub-Gaussian heat kernel estimate. That is, the small-time behaviour of the heat kernel is Gaussian as in Euclidean spaces, while the heat kernel has a subGaussian decay for large time. Fractal manifolds are typical examples satisfying these assumptions. We prove that the quasi Riesz transform is of weak type $(1,1)$.

In Chapter 4, we work on the discrete setting. Considering graphs, we prove analogous results under the corresponding assumptions to those in the Riemannian manifold setting. Note that compared with the Riemannian manifold setting, there are no heat kernel estimate and local Riesz transform.

The second subject of the thesis is the Hardy space theory. The study of real Hardy spaces was mainly developed in the 1960s and 1970s and a key paper is Fefferman and Stein [7]. Later, Coifman and Weiss [4] developed the Hardy space theory on spaces of homogeneous type. There are several different characterisations for Hardy spaces, which involve suitable maximal functions, atomic decompositions, molecular decompositions, singular integrals and so on. The theory of $H^{p}$ spaces is a very important subject in classical analysis, and these spaces appear to be natural substitutes of Lebesgue spaces. On metric measure spaces, one can extend the Hardy space theory to the context of semigroups of operators. Much recent work has been devoted to this topic (see for example [2,8]).

In Chapter 3, we develop a Hardy space theory on metric measure spaces satisfying different local and global heat kernel estimates. Let $(M, d, \mu)$ be a noncompact metric measure space with the doubling volume properties and $L$ be a nonnegative self-adjoint operator on $M$. Assume that $\left(e^{-t L}\right)_{t>0}$ satisfies an $L^{p_{0}}-L^{p_{0}^{\prime}}\left(1 \leq p_{0}<2\right)$ off-diagonal estimate with different local and global behaviour. Firstly we define Hardy spaces via molecules and square functions which are adapted to the heat kernel estimates. Then we show that the two $H^{1}$ spaces via molecules and via square functions are the same. Also, we compare the $H^{p}$ space defined via square functions with $L^{p}$. The corresponding $H^{p}$ space defined via square functions is equivalent to the Lebesgue space $L^{p}$ for $p_{0}<p<p_{0}^{\prime}$. However, it is shown that in this situation the $H^{p}$ space corresponding to Gaussian estimates may not coincide with $L^{p}$ and counter-examples are given. Finally, as an application of this Hardy space theory, we prove the $H^{1}-L^{1}$ boundedness of quasi Riesz transforms on Riemannian manifolds as in Chapter 2.

Besides the two main subjects, we also study some inequalities on Vicsek graphs. In Chapter 5, we prove generalised Poincaré inequalities and Sobolev inequalities on Vicsek graphs and we show that they are optimal. These inequalities are closely related to the Riesz transform problem and other problems in analysis.

\section{References}

[1] P. Auscher, T. Coulhon, X. T. Duong and S. Hofmann, 'Riesz transform on manifolds and heat kernel regularity', Ann. Sci. Éc. Norm. Supér. (4) 37(6) (2004), 911-957. 
[2] P. Auscher, A. McIntosh and E. Russ, 'Hardy spaces of differential forms on Riemannian manifolds', J. Geom. Anal. 18(1) (2008), 192-248.

[3] M. Barlow, T. Coulhon and A. Grigor'yan, 'Brownian motion and harmonic analysis on Sierpinski carpets', Invent. Math. 144(3) (2001), 609-649.

[4] R. Coifman and G. Weiss, 'Extensions of Hardy spaces and their use in analysis', Bull. Amer. Math. Soc. 83(4) (1977), 569-645.

[5] T. Coulhon and X. T. Duong, 'Riesz transforms for $1 \leq p \leq 2$ ', Trans. Amer. Math. Soc. 351(3) (1999), 1151-1169.

[6] T. Coulhon and X. T. Duong, 'Riesz transform and related inequalities on noncompact Riemannian manifolds', Comm. Pure Appl. Math. 56(12) (2003), 1728-1751.

[7] C. Fefferman and E. M. Stein, ' $H^{p}$ spaces of several variables', Acta Math. 129(3-4) (1972), 137-193.

[8] S. Hofmann, G. Lu, D. Mitrea, M. Mitrea and L. Yan, 'Hardy spaces associated to non-negative selfadjoint operators satisfying Davies-Gaffney estimates', Mem. Amer. Math. Soc. 214(1007) (2011), vi+78.

[9] R. S. Strichartz, 'Analysis of the Laplacian on the complete Riemannian manifold', J. Funct. Anal. 52(1) (1983), 48-79.

LI CHEN, Instituto de Ciencias Matemáticas, CSIC-UAM-UC3M-UCM, Consejo Superior de Investigaciones Científicas, C/Nicolás Cabrera

13-15, E-28049 Madrid, Spain

e-mail: li.chen@icmat.es 